\title{
Segmental ileal dilatation with supernumerary intestinal muscle coat in a neonate
}

\author{
Tutku Soyer $^{1 *}$, Beril Talim² and Feridun Cahit Tanyel ${ }^{1}$
}

\begin{abstract}
Segmental intestinal dilatation (SID) is a rare developmental anomaly of the midgut, characterized by sharply demarcated dilatation of a gastrointestinal segment with clinical findings of intestinal obstruction. Although morphologic criteria for SID are clearly delineated, etiological origin of dilated intestine is unknown. Histology of the resected segment is usually reported to have normal presence of ganglion cells in the myenteric and submucosal plexuses. Intestinal muscle is hypertrophied, and heterotopic gastric mucosa may also be encountered. A 3-day-old male infant presenting with clinical findings of intestinal obstruction was diagnosed to have SID and had supernumerary intestinal muscle coat (SIMC) in the dilated segment, without any evidence of neurological abnormality. Histopathological findings of the resected ileal segment are reported to discuss the role of architectural malformation of muscularis propria as a cause of SID.
\end{abstract}

Keywords: Segmental ileal dilatation; Neonate; Gastrointestinal smooth muscle

\section{Background}

Segmental intestinal dilatation (SID) is a rare gastrointestinal anomaly introduced by Rossi and Giocomoni [1]. According to the criteria proposed by Swenson and Rathauser, SID is defined as limited bowel dilatation with three- to fourfold increased size, with an abrupt transition between dilated and normal bowel, without any intrinsic and extrinsic barrier distal to the dilatation or abnormal neurological innervation [2]. Approximately half of the SID cases are seen in the first days of life, and clinical findings of intestinal obstruction are the most common presentation [3]. Anemia, failure to thrive, and abdominal pain are usually encountered in older children [3].

Although diagnostic criteria of SID are well established, the etiology of the disease is unclear. Histology of the involved bowel usually shows normal neurological innervation with a hypertrophied or thin muscle layer [4]. Heterotopic esophageal and gastric mucosa or cartilaginous foci are also reported. However, none of the histopathological findings are able to explain the etiology of SID.

\footnotetext{
* Correspondence: soyer.tutku@gmail.com

'Department of Pediatric Surgery, Faculty of Medicine, Hacettepe University, 06100 Ankara, Turkey

Full list of author information is available at the end of the article
}

The presence of an additional smooth muscle layer in the intestine is a rare congenital anomaly of enteric smooth muscle and considered as a cause of pseudoobstruction or constipation [5]. Supernumerary intestinal muscle coat (SIMC) has been reported in patients with Hirschsprung disease and intestinal pseudo-obstruction. However, it has not been identified in the resected bowel specimens of patients with SID previously.

A 3-day-old male infant with intestinal obstruction was diagnosed as SID, and the patient underwent resection of involved bowel and anastomosis. Microscopical evaluation of the resected segment revealed SIMC with normal neurological innervation. We aim to discuss the role of architectural malformation of intestinal muscle coat in the etiology of SID.

\section{Case presentation}

A 20-day-old male infant, weighing 3,200 g, was delivered vaginally at 37 weeks of gestation. He was admitted to the emergency department with abdominal distention and bilious vomiting. He had inability to pass stool in the first 3 days of his life. Then, he irregularly passed the stools with rectal stimulation. His prenatal history was unremarkable. He has no family history of pseudointestinal obstruction. 
On admission, his height and weight were at 50th percentile, axillary temperature was $37^{\circ} \mathrm{C}$, pulse was 130/bpm, and blood pressure was 60/45 mmHg. Physical examination was normal except a distended abdomen. Laboratory findings revealed hemoglobin $13.5 \mathrm{~g} / \mathrm{dL}$, hematocrit $33.2 \%$, leukocyte $10.2 \times 10^{3} / \mu \mathrm{L}$, and platelet $310 \times 10^{3} / \mu \mathrm{L}$. Biochemical analyses including liver and renal function tests were within normal limits. The physical examination of all systems was normal except abdominal distention. His abdominal X-ray showed a distended large bowel loop with air-fluid levels (Figure 1). He underwent colon contrast enema with presumptive diagnosis of Hirschsprung disease and was diagnosed as segmental dilatation of ileum with normal colon (Figure 2). In surgical exploration, a dilated ileal segment of $15 \mathrm{~cm}$ was detected $25 \mathrm{~cm}$ distal to the duodenum and $30 \mathrm{~cm}$ proximal to the terminal ileum. This segment was fivefold dilated, and neither extrinsic nor intrinsic barrier was found as a cause of the dilatation (Figure 3). The dilated segment of the ileum was resected, and the bowel continuity was achieved by end-to-end anastomosis. During exploration, the cecum was found at the left upper quadrant, and malrotation of intestines was detected. Ladd bands between the duodenum and colon were excised, and Ladd procedure was completed with appendectomy.

Macroscopic examination of the $15-\mathrm{cm}$ resection specimen revealed a dilated segment of $10 \mathrm{~cm}$ in length and $4 \mathrm{~cm}$ in diameter with sudden transition to normal intestinal diameter on both sides. Mucosal surface was intact. Microscopic evaluation of the non-dilated intestinal segment showed normal histology with inner circular

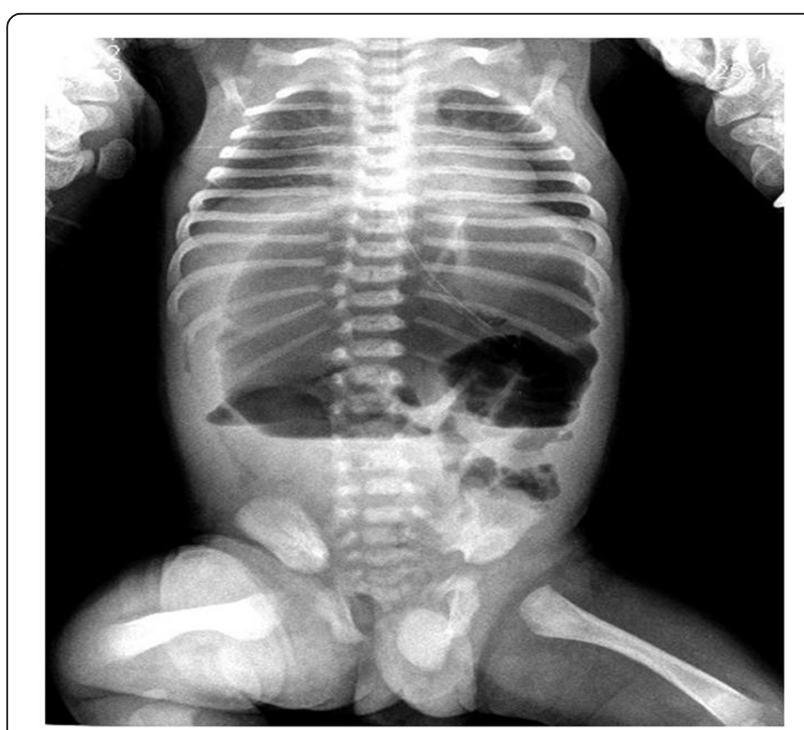

Figure 1 Abdominal X-ray showing a distended large bowel loop with air-fluid levels.

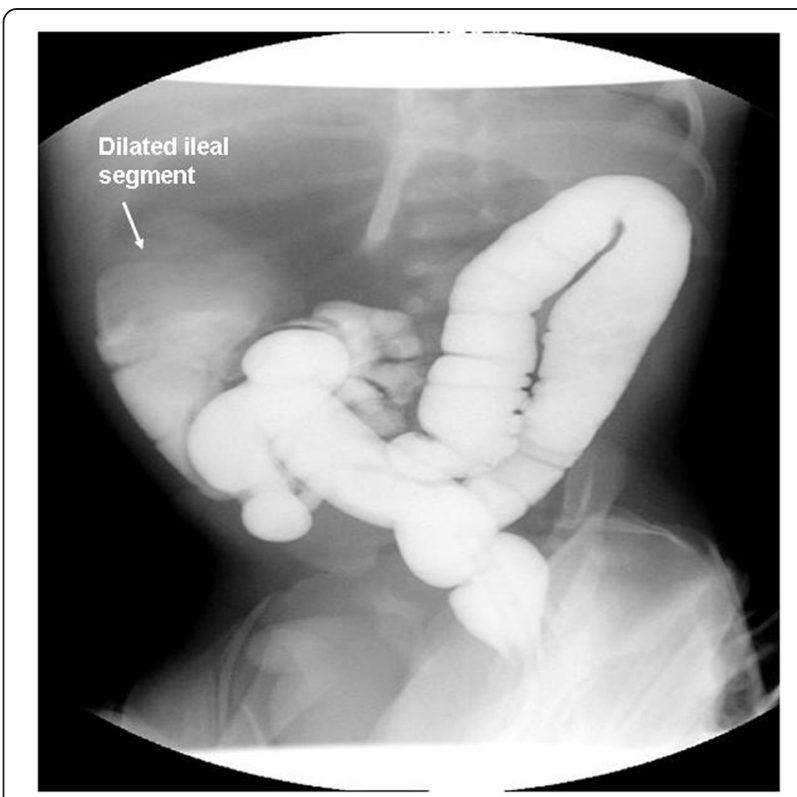

Figure 2 Contrast enema showing segmental dilatation of the ileum with normal colon.

and outer longitudinal smooth muscle layers of muscularis propria with normally distributed myenteric neural elements and ganglion cells and interstitial cells of Cajal, as shown by c-kit (CD117) immunohistochemical staining (Figure 4). In the sections from the dilated segment, in addition to the unremarkable inner circular smooth muscle and the adjacent longitudinal layer, an extra smooth muscle layer was present in the outermost side of muscularis propria, next to serosa (Figure 5). This additional external smooth muscle coat showed irregular thickness and haphazard orientation, mostly longitudinally

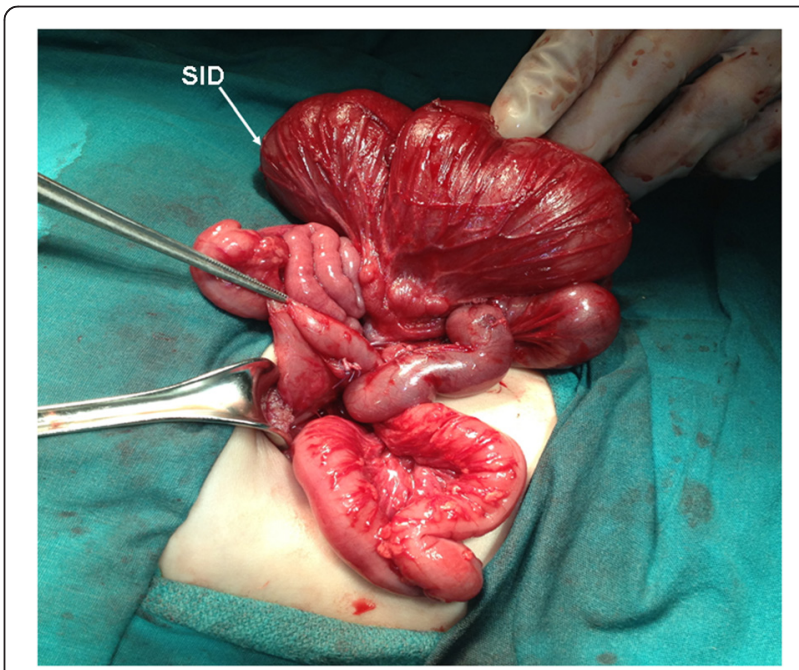

Figure 3 Fivefold dilated ileal segment with malrotation. 


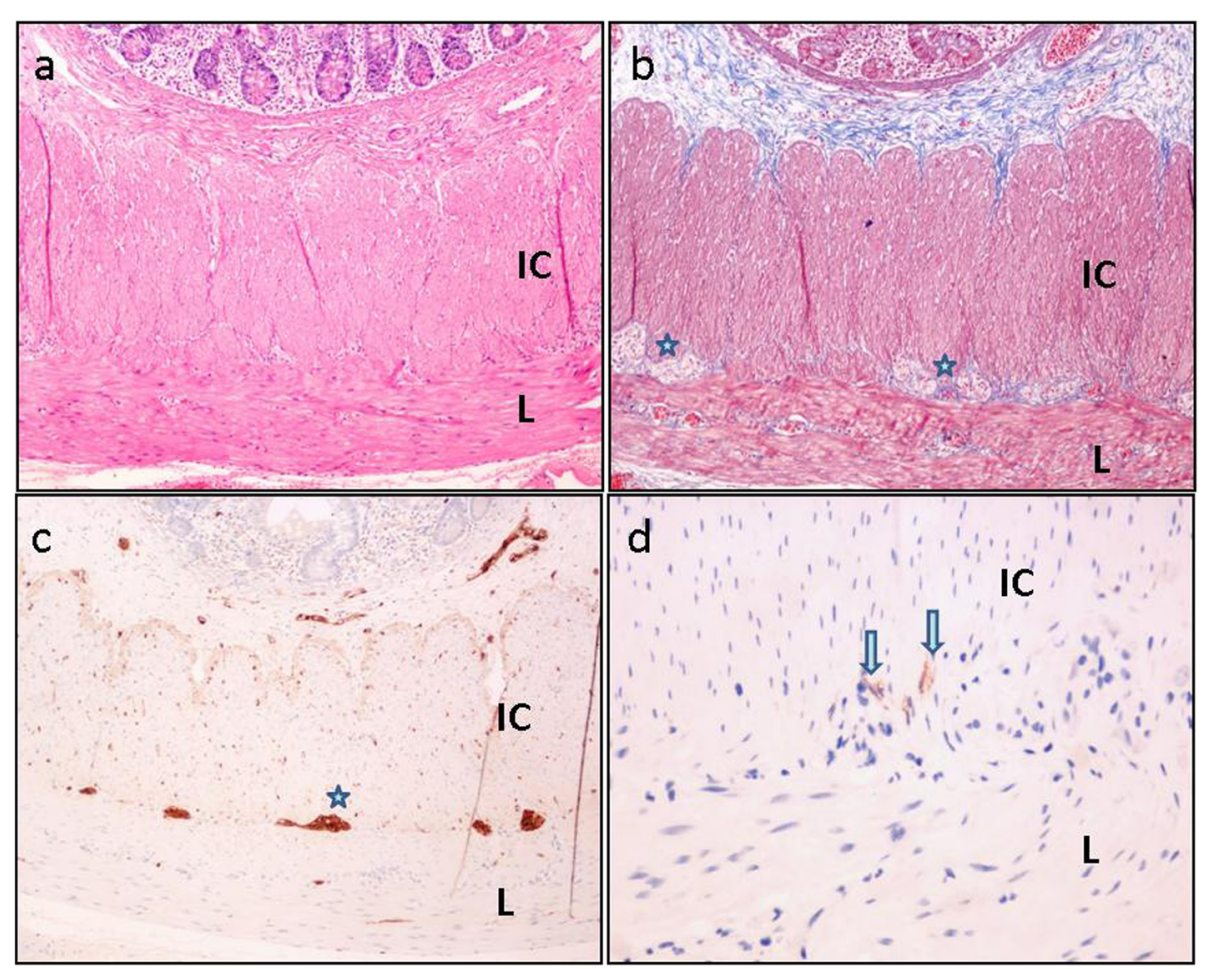

Figure 4 Microscopic evaluation of the non-dilated intestinal segment. Sections from the non-dilated segment showing normal architecture consisting of inner circular (IC) and outer longitudinal (L) smooth muscle layers. Ganglia and nerves (stars) as well as interstitial cells of Cajal (arrows) are non-remarkable. (a) Hematoxylin and eosin (H\&E), (b) Masson trichrome, (c) S-100, and (d) CD117 (c-kit). (a-c) Original magnification $\times 10$. (d) Original magnification $\times 40$.

arranged but showing oblique fascicles in some areas. Myenteric plexus and ganglion cells were normally placed between the inner circular and longitudinal muscle layers but were also present between the longitudinal and the outermost extracircular smooth muscle layers (Figure 5b). Interstitial cells of Cajal were detected on both sides of the longitudinal muscle (Figure $5 \mathrm{c}, \mathrm{d}$ ). There was no vacuolar change in the smooth muscle layer. Mucosa was unremarkable. The appendix was normal histologically. He was fed orally at the fifth postoperative day and discharged from the hospital after an uneventful period of 8 days. He has been followed up for the last 8 months, and he is free of symptoms.

\section{Discussion}

SID is a rare congenital anomaly characterized by sharply demarcated dilatation of gastrointestinal tract without any evidence of mechanical obstruction [3]. The most common localization of dilated segment is the ileum and followed by the colon, jejunum, and duodenum [6,7]. Approximately half of the SIDs present in the first day of life [8]. Rarely, some cases become symptomatic during infancy and adolescence. SID is encountered in both sexes with a slight male predominance [3]. The most common clinical presentation is intestinal obstruction, and abdominal pain, anemia, and failure to thrive can be seen in older children [3]. Anemia is the first sign of SID in patients with heterotopic gastric mucosa. It has been reported that one third of SID cases have been detected incidentally during laparotomy [8]. Omphalomesenteric remnants and malrotation are reported as associated anomalies in SID. Malrotation associated with SID was also recognized in our patient during laparotomy.

Although diagnostic criteria are well established, the etiology of the disease is unclear. It has been suggested that transient embryonic structures such as vitelline vessels and omphalomesenteric bands cause extrinsic compression of both ends of bowel [9,10]. In addition, in utero vascular accidents, primary aplasia of an intestinal segment, and congenital damage to the nerves in myenteric plexus are considered as possible causes of SID [11]. The role of enteric nervous system and interstitial cells of Cajal was also investigated, and no neurogenic etiology could be attributed to SID [12]. We have also detected normal distribution of interstitial cells of Cajal in our case.

SID can also be detected as an intra-abdominal cystic mass in prenatal ultrasonography [13]. On abdominal $\mathrm{X}$-ray, it is associated with dilated bowel loops with 


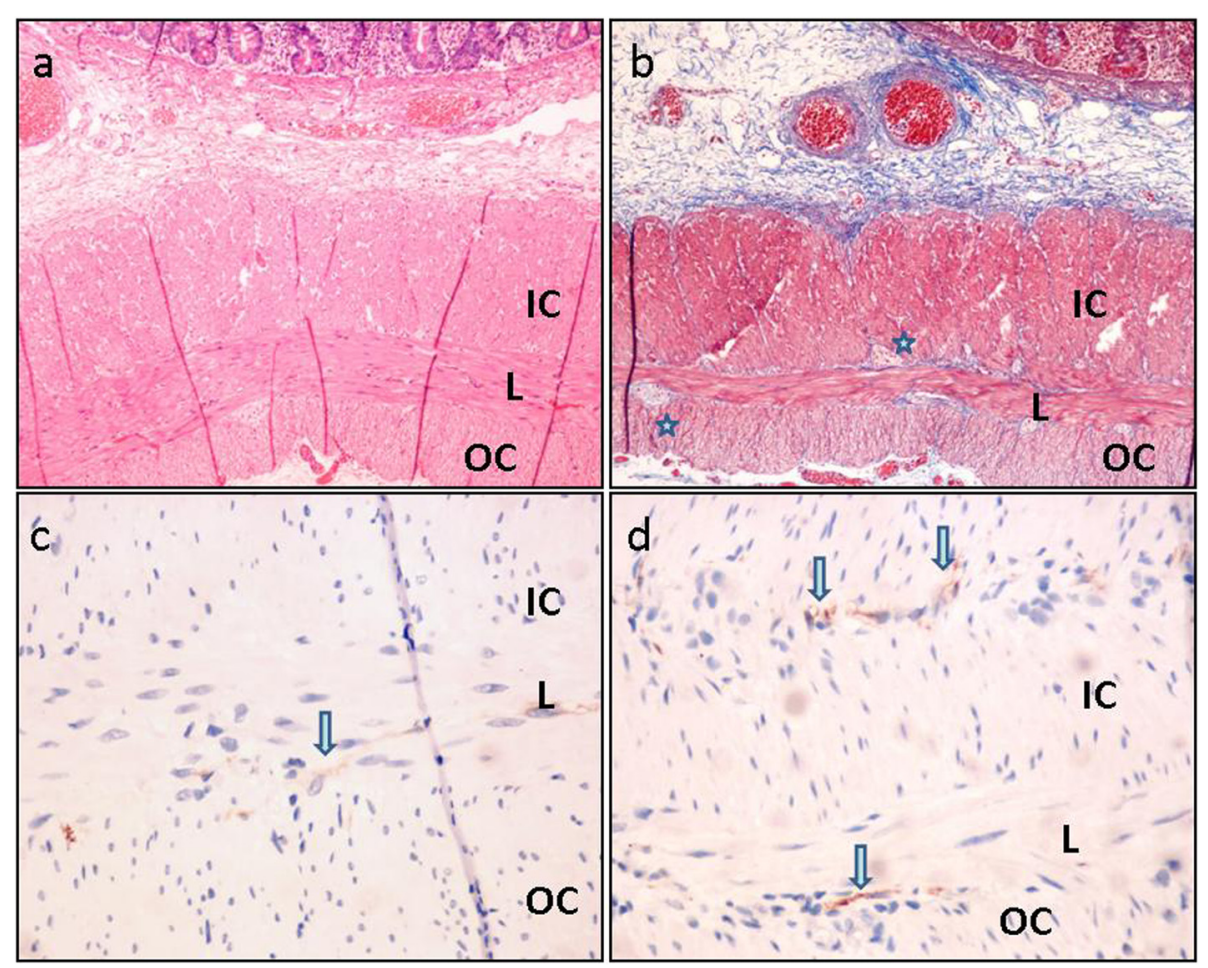

Figure 5 Sections from the dilated segment. In addition to the inner circular (IC) and longitudinal (L) layers, there is an extra outer smooth muscle layer (OC). Ganglia and nerves (stars) (b) and interstitial cells of Cajal (arrows) (c, d) are located on both sides of the longitudinal muscle. (a) Hematoxylin and eosin (H\&E), (b) Masson trichrome, and (c-d) CD117 (c-kit). (a-b) Original magnification $\times 10$. (c-d) Original magnification $\times 40$.

or without air-fluid levels and can be easily misdiagnosed as other causes of intestinal obstruction [3]. As in our case, contrast enema studies can be performed to differentiate other gastrointestinal anomalies. Contrast enema studies show marked segmental dilatation of small bowel loop with normal colon findings. Since most of the neonates undergo contrast enema studies, the use of computed tomography in the diagnosis of SID is limited and reserved for older patients.

The treatment of SID is resection of dilated segment and end-to-end anastomosis [3]. Most patients have uneventful course, and prognosis is excellent after surgical resection.

In most of the cases, histology of the resected segment is usually normal [3]. However, some of the cases showed hypertrophied or very thin muscle layer in the involved segment in histopathological evaluation. Heterotopic esophageal or gastric mucosa or cartilaginous foci are also reported in dilated intestinal segment [3]. Kobayashi et al. suggested that ectopic gastric mucosa causes interruption in the neural and muscular network in the dilated intestine [14]. However, all resected segments show normal presence of ganglion cells in myenteric and submucosal plexuses [6]. Histopathological recognition of localized vacuolization of the smooth muscle layer has suggested that myopathy might contribute to the pathogenesis of SID [6]. However, there are no reports of additional smooth muscle layer in the involved segment of SID in the literature. In the case reported here, no mucosal abnormality was present, ganglia and plexuses were normal in distribution, and there was no vacuolization in smooth muscle layer.

Architectural malformations of muscularis propria consist of supernumerary and/or haphazard orientation of muscle fibers with an additional neural plexus. Smith et al. have described supernumerary muscle coat in five patients and classified them as diffuse or segmental [15]. SIMC is also reported in a patient with Hirschsprung disease and considered as a congenital anomaly rather than an acquired histopathological alteration [5]. Xlinked intestinal pseudo-obstruction due to filamin A mutation should be also considered in the differential diagnosis of SMIC. Kapur et al. reported FLNA mutations with intestinal pseudo-obstruction. They usually show male predominance, short small intestinal length, and malrotation [16]. FLNA mutations include abnormal intestinal layering throughout a longer segment of the intestine with neurologic impairment in histology. In our patient, abnormal layering is limited to a short segment of the intestine, with normal ganglia and without associated neurologic disease. In our case with SID, we have found segmental SIMC in the dilated segment. 
There was no vacuolar change in the muscle layers, but haphazard orientation was remarkable in some areas. Myenteric plexuses and ganglion cells were normally placed between the inner circular layer and the longitudinal layer, but some plexuses were also present between the longitudinal layer and the outermost circular extra smooth muscle layer. Although the role of myopathy was suggested in the etiology of SID, SIMC as a cause of SID has not been reported previously. We suggest that hypertrophied muscle or very thin muscle layer should be considered as result rather than the cause of SID [10]. SIMC or architectural malformation of the intestinal smooth muscle should be considered as the most likely cause of segmental dilatation. Presence of myenteric plexuses and ganglion cells towards the external surface also supports that this is a primary developmental problem.

\section{Conclusions}

SID is a congenital anomaly of gastrointestinal tract with clinical findings of intestinal obstruction. We show for the first time that at an extremely rare malformation of muscularis propria, SIMC can be seen in the dilated segment of SID, and we suggest that this additional muscle layer can play a role in the etiology of SID.

\section{Consent}

Written informed consent was obtained from the patient for publication of this case report and any accompanying images. A copy of the written consent is available for review by the Editor-in-Chief of this journal.

\section{Abbreviations}

SID: segmental ileal dilatation; SMIC: supernumerary intestinal muscle coat.

\section{Competing interests}

The authors declare that they have no competing interests.

\section{Authors' contributions}

TS has made substantial contributions to the conception and design, acquisition of data, or analysis and interpretation of data. BT has made substantial contributions to the conception and design, acquisition of data, or analysis and interpretation of data. FCT has been involved in the drafting of the manuscript or revising it critically for important intellectual content.

All authors read and approved the final manuscript.

\section{Author details}

'Department of Pediatric Surgery, Faculty of Medicine, Hacettepe University, 06100 Ankara, Turkey. ${ }^{2}$ Department of Pediatrics, Pathology Unit, Faculty of Medicine, Hacettepe University, Ankara, Turkey.

Received: 1 November 2014 Accepted: 20 January 2015

Published online: 17 February 2015

\section{References}

1. Rossi R, Giacomoni MA. Segmental dilatation of the jejunum. J Pediatr Surg. 1973:8:335-6.

2. Swenson O, Rothauser F. Segmental dilatation of the colon: a new entity. Am J Surg. 1959;97:734-8.

3. Paradiso FV, Coletta R, Olivieri C, Briganti V, Oriolo L, Fabbri R, et al. Antenatal ultrasonographic features associated with segmental small bowel dilatation: an unusual neonatal condition mimicking congenital small bowel obstruction. Pediatr Neonatol. 2013;54:339-43.

4. Ueda T, Okamato E. Segmental dilatation of the ileum: report of a case. J Pediatr Surg. 1972;7:292-3.

5. Leong M, Verey F, Newbury-Ecob R, Ramani P. Supernumerary intestinal muscle coat in a patient with Hirschsprung disease/Mowat-Wilson syndrome. Pediatr Dev Pathol. 2010;13:415-8.

6. Saha S, Konar H, Chatterjee P, Basu KS, Chatterjee N, Thakur SB, et al. Segmental ileal obstruction in neonates-a rare entity. J Pediatr Surg. 2009;44:1827-30

7. Katsura S, Kudo T, Enoki T, Taguchi T, Hamano K. Congenital segmental dilatation of the duodenum: report of a case. Surg Today. 2011;41(3):406-8

8. Eradi B, Menon P, Rao KL, Thapa BR, Nagi B. Segmental dilatation of ileum: an unusual cause of severe malnutrition. Pediatr Surg Int. 2005;21:405-6.

9. Morewood DJ, Cunningham ME. Case report: segmental dilatation of ileum presenting with anaemia. Clin Radiol. 1985;36:267-8.

10. Kuint J, Avigad I, Husar M, Linder N, Reichman B. Segmental dilatation of ileum: uncommon cause of neonatal intestinal obstruction. J Pediatr Surg. 1993:28:1637-9.

11. Ratan SK, Kulsreshtha R, Ratan J. Cystic duplication of the cecum with segmental dilatation of the ileum: report of a case. Surg Today. 2010;31:72-5.

12. Cheng W, Lui VC, Chen QM, Tam PK. Enteric nervous system, interstitial cells of Cajal and smooth muscle vacuolization in segmental dilatation of jejunum. J Pediatr Surg. 2001;36:930-5.

13. Park JS, Doh HJ, Park ES, Seo JH, Lim JY, Park CH, et al. Segmental dilatation of the ileum presenting as a cystic lesion on prenatal ultrasonography in one twin. Pediatr Int. 2010;52:337-8.

14. Kobayashi T, Uchida N, Shiojima M, Sasamoto H, Shimura T, Takahasi A, et al. Segmental dilatation of the ileum covered almost entirely by gastric mucosa: report of a case. Surg Today. 2007;37:1102-4.

15. Smith W, Milla PJ. Histological phenotypes of enteric smooth muscle disease causing functional intestinal obstruction in childhood. Histopathology. 1997;31:112-22.

16. Kapur RP, Robertson SP, Hannibal MC, Finn LS, Morgan T, von Kogelenberg $\mathrm{M}$, et al. Diffuse abnormal layering of small intestinal smooth muscle is present in patients with FLNA mutations and X-linked intestinal pseudoobstruction. Am J Surg Pathol. 2010;34:1528-43.

\section{Submit your manuscript to a SpringerOpen ${ }^{\odot}$ journal and benefit from:}

- Convenient online submission

- Rigorous peer review

- Immediate publication on acceptance

- Open access: articles freely available online

- High visibility within the field

- Retaining the copyright to your article

Submit your next manuscript at $>$ springeropen.com 